\title{
Comparative Study of Band-Power Extraction Techniques for Motor Imagery Classification
}

\author{
Nicolas Brodu \\ LTSI, University of Rennes 1 \\ Campus de Beaulieu, Bât 22 \\ 35042 Rennes Cedex - France \\ Email: nicolas.brodu@univ-rennes1.fr
}

\author{
Fabien Lotte \\ Institute for Infocomm Research (I2R) \\ Signal Processing Department \\ Brain-Computer Interface Laboratory \\ 1 Fusionopolis way, 138632, Singapore \\ Email: fabien.lotte@gmail.com
}

\author{
Anatole Lécuyer \\ INRIA \\ Campus de Beaulieu \\ Avenue du Général Leclerc \\ 35042 Rennes Cedex - France \\ Email: anatole.lecuyer@inria.fr
}

\begin{abstract}
We review different techniques for extracting the power information contained in frequency bands in the context of electroencephalography (EEG) based Brain-Computer Interfaces (BCI). In this domain it is common to apply only one algorithm for extracting the power information. However previous work and our current study confirm that one may indeed expect varying degrees of success by choosing inadequate algorithms for the power extraction. Our results suggest that on average one algorithm seems superior for extracting the power information for Motor Imagery tasks : the application of a Morlet wavelet on the raw EEG signals, with the time-frequency resolution tradeoff selected by cross-validation.
\end{abstract}

\section{INTRODUCTION}

This paper proposes a comparative study of various techniques for estimating the power information contained in different frequency bands of a real signal, in the specific context of motor imagery (i.e., limb movement imagination) for Brain-Computer Interfaces (BCI) [1]. The specificity of the BCI field is to deal with very noisy data captured by Electro-Encephalo-Graphy (EEG). The goal is to infer the user's mental state from his/her EEG signals. In a BCI setup we consider in this study the task is to find whether the user imagines left hand or right hand movements, in order to build an interactive Human-Computer interface based only on brain activity. Practical applications are envisionned for severly disabled people who cannot move their limbs but whose brain is still functionnal [2].

One major challenge of this field is thus to extract reliable information from noisy data in real time in the form of relevant features [3]. These can then be passed on to classification techniques for identifying the user's mental state. Physiological arguments suggest that the $\mu$ and $\beta$ frequency bands (around 8$12 \mathrm{~Hz}$ and $16-24 \mathrm{~Hz}$ ) are especially relevant for discriminating motor activity. A common approach in the BCI field is thus to extract the power information from the signal over these frequency bands and use that as the classification feature.

There is a wide litterature on power estimation (see [4] for pointers). Time-frequency decomposition is in itself a wide topic of research, which has been extensively studied. Different techniques allow a practitionner to select the best trade-off to make between time and frequency resolutions, the presence of artifacts (spectral bias) or increased precision, etc. Yet most BCI framework only propose or consider a few of these techniques and with a limited range of trade-offs. A thorough comparison of different power feature extraction techniques in the context of BCI was presented in [5], together with their use in classification of motor imagery. This paper aims at complementing and reproducing independently the observations made in that survey. Notable differences between this paper and [5] include:

- Eleven out of the 13 subjets we used for our benchmark are distinct from [5]. We used a subset of the most representative power estimation techniques based on their results and as justified in the next section.

- While some of our results confirmed independently the results obtained in [5], other contrasted them. More particularly, we showed that when the time-frequency resolution is properly selected the Morlet wavelet can be the most efficient band-power extraction technique, whereas it was reported the less efficient one in [5].

- In [5] the power density is extracted along each timefrequency decomposition atom (See Fig 1), and the energy is summed over frequency components at that time. In this paper we instead prioritise the frequency representation and sum the energy over the trial duration. This is justified physiologically by considering that motor imagery related brain activity is more easily discriminable in the frequency domain than in the time domain (i.e. we are looking for frequency signatures rather than time patterns). Moreover, focusing on a frequential signature rather than on a temporal signature is a popular and efficient approach that has been used and validated by several leading BCI laboratories such as the Graz BCI or the Berlin BCI [6], [7], [8].

- We use the log-transform of the energy in each frequency band as the discriminant feature. We decided to compute the energy on $1 \mathrm{~Hz}$ bands from 4 to $30 \mathrm{~Hz}$, for both the $\mathrm{C} 3$ and $\mathrm{C} 4$ electrodes. One can recover the physiological $\mu$ and $\beta$ bands (around $8-12 \mathrm{~Hz}$ and $16-24 \mathrm{~Hz}$ ) by combining the corresponding $1 \mathrm{~Hz}$ bands to reduce the number of features, which was done in [5]. Yet we prefer to let the classifiers exploit all available information rather 
than fixing the $\mu$ and $\beta$ bands a priori. This is indeed justified by the well known inter-subject variability in motor-imagery based BCI [9].

- We used only the Linear Discriminant Analysis (LDA) classifier as it is well-used in the BCI litterature [10]. In [5], a genetic algorithm had to be used to fine-tune Support Vector Machines. But we want to assess the performance of the extraction methods. Using a nonparametric classifier like LDA offers a stable basis of comparison and avoids any interference from the SVM training procedure.

In the next section we detail how we extracted the features and present the corresponding techniques we used to extract the band-power information. Section III presents the data set we used and the results of our experiments. The paper ends with a conclusion and some recommendations based on our results.

\section{POWER IN FREQUENCY BANDS}

The next subsections describe each band-power estimation technique we used and how they were extracted in the present study. We considered using the periodogram, the spectrogram and the Morlet wavelet scalogram to cover a diversity of timefrequency samplings (see Fig 1); the Wigner-Ville distribution following [4] because it represents an alternative compromise to the spectrogram (see Section II-B); the power spectral density estimation using an auto-regressive model with the Yule-Walker algorithm for its good results in [5] and because it brings another way of estimating the spectrum compared to the aformentioned approaches; and the Butterworth filter since it is a commonly used feature in the BCI litterature [1], [11].

\section{A. The spectrogram method}

A usual way to extract the power in frequency bands is to use the spectrogram method [4] to get a time-frequency signal decomposition:

- The signal is convolved with a windowing function that is localised in time around $t$. A Fourier transform is then performed on the convolved signal, non null only on a sliding chunk centred on $t$, leading to a time-frequency representation $X(f, t)$ of the original signal $x(t)$ (see Fig 1 , second case).

- The energy marginals in the frequency domain [12] allow us to estimate a spectrum: $E_{f}=\sum_{t}|X(f, t)|^{2}$

- The energy in each band $B$ is obtained by summing the marginals in that band: $E_{B}=\sum_{f \in B} E_{f}$.

As explained in the introduction, a feature vector is then formed from the logarithms of the estimated energy in each $1 \mathrm{~Hz}$ band.

\section{B. The Wigner-Ville distribution}

As shown in [4] there is a trade-off to make between increased precision for the energy estimation in each decomposition region of the time-frequency plane (see Fig 1), and the occurrence of artifacts. These artifacts denote alterations due to the frequency decomposition and not physiological artifacts such as EOG or EMG. The spectrogram introduces little artifacts, and the Wigner-Ville distribution achieves the opposite trade-off: improved accuracy over each decomposition atom, at the cost of more artifacts.

An hypothesis is that the improved localisation in frequency could compensate the presence of the artifacts for the classification tasks.

\section{The Morlet wavelet scalogram}

The scalogram is the third splicing of the time-frequency plane represented on Fig 1. A mother wavelet $\psi(x)$ function is scaled and shifted and forms a basis for the decomposition. The frequency interpretation comes from the fact that if the mother wavelet is localised at a given frequency $f_{0}$, then a scaled version $\psi(a x)$ is localised at frequency $\frac{f_{0}}{a}$ by the scaling property of the Fourier transform. The wavelet decomposition formula can thus be rewritten as we did in Fig. 1 to highlight $\frac{f}{f_{0}}$ as a scaling factor.

In [5] the mother wavelet is carefully chosen so the relevant $\mu$ and $\beta$ bands are well covered in the time-frequency plane, since they combine the energy contributions in these bands into a single feature. In the present study we use the facility provided by [12] to adapt the wavelet so that $f=\frac{f_{0}}{a}$ ranges between 4 and $30 \mathrm{~Hz}$ : For a Morlet wavelet $f_{0}$ can be chosen arbitrarily, and the scale parameter $a$ is adapted to maintain the selected frequency range. The influence of this free parameter however is to shape the effective resolutions on both axis in the time-frequency plane as shown in 1 . In the present study we use a 10-fold cross-validation on the training set in order to select the best time-frequency resolution compromise.

\section{Periodogram on the whole signal}

The spectrogram method considered sliding chunks because it is primarily used for time-frequency decomposition: the energy is localised with a fixed resolution in both the frequency and time domain. However here we only perform a classification step at the end of trials. Thus we are only interested in the frequency components over a fixed signal duration, not at each instant. Applying an FFT transform to the whole signal and proceeding directly to the power estimation better matches the structure of the time-frequency decomposition we need, as depicted in Fig. 1. Technically the periodogram is thus not a time-frequency decomposition method, only a frequency decomposition on the whole signal.

Unfortunately as the signal is usually finite and non-periodic this technique introduces spurious artifacts in otherwise unrelated frequency bands (known as spectral bias). A workaround is to apply a window function on the whole signal, but we loose some information as the window would smoothly damp it to 0 on each end. The spectrogram copes with this by sliding each chunk in time, thus getting a correct estimate over each chunk centre.

A second workaround is to remove the 0 and low-frequency components so as to detrend the signal: This operation centres the signal around 0 , and the periodisation implicitly operated by the FFT results in less artifacts. We applied this second 
Fourier transform

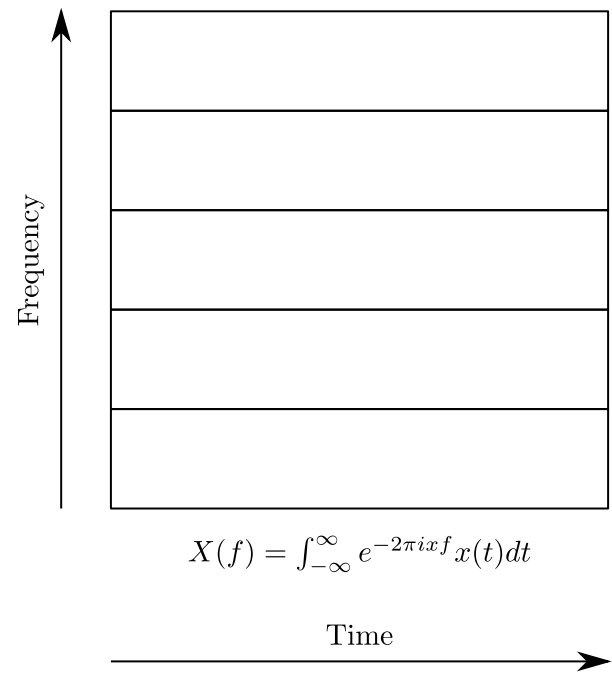

Short-time (windowed) Fourier transfom

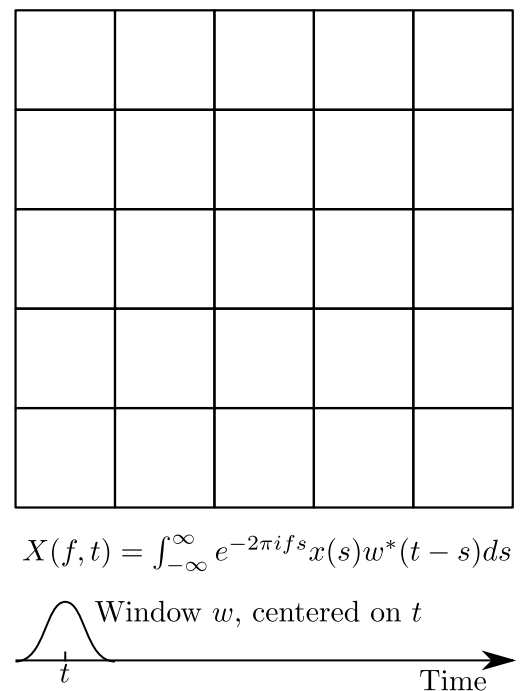

Wavelet scalogram

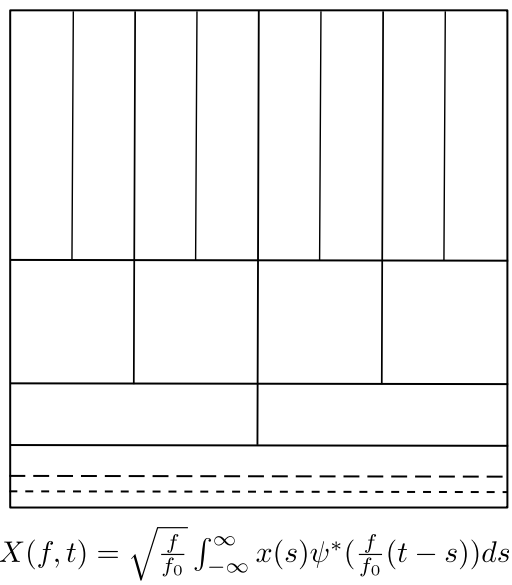

Mother wavelet $\psi$ 's main frequency: $f_{0}$

Time

Figure 1. Splicing the time-frequency plane

workaround by pre-filtering the signals with a linear phaseresponse high-pass FIR filter: at least $50 \mathrm{~dB}$ attenuation at $0 \mathrm{~Hz}$ and less than $1 \mathrm{~dB}$ change at $4 \mathrm{~Hz}$ in order to preserve the physiologically relevant bands.

The periodogram is then simply computed:

- The signal $x(t)$ is taken as a whole, without applying a window function, but filtered as aforementioned.

- A Discrete Fourier Transform is applied, giving a frequency representation $X(f)$ of the whole signal, including artifacts due to the non-periodic nature of the signal (attenuated by the FIR pre-filtering).

- Power is extracted in narrow bands $B$ (of $1 \mathrm{~Hz}$ in this example): $E_{B}(X)=\sum_{f \in B}|X(f)|^{2}$.

\section{E. Band-pass filtering with a Butterworth IIR filter}

It is also possible to directly apply a band-pass filter to the signal $x(t)$ in order to damp out frequencies outside each selected band $B$ : the resulting filtered signal $y(t)$ presumably contains only the selected frequencies. Assuming a perfect block filter, it is then possible to estimate the energy in these bands by squaring the magnitude of the time series samples directly: $E_{\text {band }}=\sum_{t}|y(t)|^{2}$.

This is a frequent feature found in the BCI field [1]. This is the method that is performed by default in the OpenViBE software [11], with a Butterworth IIR filter of order 4, and that was also used for [13].

In the present study we select the order of the Butterworth filter on the training data set in a 10-fold cross-validation step.

\section{F. Power spectral density estimation using an auto-regressive model}

The Yule-Walker algorithm can be used to train an autoregressive model. The power spectral density is then derived from the model's response. As in [5] we estimate the order of the auto-regressive model with a 10-fold cross-validation on the training data set (using a least-squarred error hyperplane fit as the classifier). However the feature we extract is the log-transform of the energy in each of the bands. We did not consider using directly the auto-regressive model coefficients as the feature vector as this was covered already in [5].

\section{EVALUATION}

\section{A. Data sets}

We have applied the power estimation techniques on 13 subjets. Twelve subjets are taken from the BCI competitions II, III and IV ${ }^{1}$ [14][15]. We also captured data with the software platform OpenViBE [11] $]^{2}$.

1) BCI competition data sets: These data set were captured and provided by the Department of Medical Informatics, Institute for Biomedical Engineering, University of Technology Graz [16]. All the data we used follow the same experimental protocol, with minor differences explained below. In a first step a visual clue is presented to the subjet indicating left or right. The subject shall then imagine moving the corresponding hand for a fixed duration. The results presented below estimate the power over all this imaginated period. In some experiments a visual feedback is provided to the user during this period to give him a clue as to how well the signal can be detected with an internal classifier. A few seconds separate each trial, and the whole experiment is repeated with another random left or right direction.

We used data from three installments of the competitions:

- BCI competition II, data set III: This set contains data captured from only one subjet. 280 trials were equally spread in the left and right hand class. The $\mathrm{C} 3, \mathrm{C} 4$ and $\mathrm{Cz}$ electrodes were sampled at $128 \mathrm{~Hz}$. Following [17]

\footnotetext{
${ }^{1}$ http://www.bbci.de/competition/

${ }^{2}$ http://openvibe.inria.fr/?q=datasets
} 
we used only the $\mathrm{C} 3$ and $\mathrm{C} 4$ electrodes, during the trial period $3 \mathrm{~s}$ to $9 \mathrm{~s}$.

- BCI competition III, data set IIIb. Three subjets are provided, but we used only the subjects labelled S4 and $\mathrm{X} 11$ due to errors in the data of the remaining subject ${ }^{3}$. Again we used only the C3 and C4 electrodes, which were sampled in this data set at $125 \mathrm{~Hz}$, during the presentation period $4 \mathrm{~s}$ to $7 \mathrm{~s}$.

- BCI competition IV, data set IIb. Nine subjets are provided. Although the experimental protocol remains the same the competition organisers warned against the presence of occular artefacts that are not present in the other two previous competitions. One goal of this data set was precisely to assert how well techniques can cope with the presence of the artifacts. In the current study we simply ignore them: we want to compare various ways to extract the power in frequency bands, irrespectively of whether the data contain artifacts or not. Should some power extraction techniques be less sensitive than the others to this kind of noise, then we are happy to have our benchmark include this more realistic case. The total of the visual cue and imaginary durations in the provided signals is $4 \mathrm{~s}$ or $4.5 \mathrm{~s}$ depending on the trials.

All these data sets were preprocessed by the Graz team with a band-pass filter between 0.5 and $30 \mathrm{~Hz}$. We unfortunately do not have access to the raw data for comparing directly the various power extraction techniques. We thus assume that the filter they used does not interfere too much in the bands we used for comparison: between 4 and $30 \mathrm{~Hz}$.

2) OpenViBE data set: We captured data from one subject using the same protocol as for the BCI competition experiments.

We recorded 560 trials over two weeks, equally spread on the left and right hand movement classes, each trial lasting $5 \mathrm{~s}$. We then randomly selected half the trials for training with the constraint of maintaining the same number of left and right instances. The remaining trials were used as the test set. We recorded 10 electrodes at $512 \mathrm{~Hz}: \mathrm{C} 3, \mathrm{C} 4, \mathrm{FC} 3, \mathrm{FC} 4, \mathrm{C} 5$, $\mathrm{C} 1, \mathrm{C} 2, \mathrm{C} 6, \mathrm{CP} 3, \mathrm{CP} 4$ (nasion reference). We then applied a discrete Laplacian spatial filter [18], [19] over the C3 and C4 electrodes.

\section{B. Comparison of the power extraction techniques}

Table I shows the result of classifying the signals from the test sets using each of the techniques presented in Section II with a Linear Discriminant Analysis classifier, as this is a classifier of choice with good success records in the BCI litterature [10]. The parameters described in each respective method subsection (ex: Butterworth filter length, auto-regressive model order, etc) were determined by searching the parameter space and optimising the 10-fold cross-validation accuracy on the training set.

We compare each method using the classification accuracy of the LDA classifier. In addition we also note for each subject

\footnotetext{
${ }^{3}$ See the notes at http://www.bbci.de/competition/iii/desc_IIIb_ps.html
}

which is the maximal performance obtained with any of the power-band extraction method. The difference to that maximal performance, on average, allows us to assert the performance of the power-band method itself: an ideal extraction technique would always be the best one. In a real case there are fluctuations between subjects and experimental frameworks, but a method that is better than the other on average (and reliably so, i.e. with low deviation from that average) shall still be prefered. These results are reported on the last two lines of Table I.

We also compared the influence of performing a pre-filtering on the data. Some methods indeed benefit from such prefiltering. As explained in section II-D, detrending the data by removing low-frequency components increase the periodogram accuracy by removing spectral bias. Similarly, training an auto-regressive model only on the frequencies of interest leads to better results than training it on irrelevant frequency components. On the other hand some methods like the Morlet Wavelet are intrinsically localised in time and frequency, so the pre-filtering does not necessary help in this case. Morevover the filters we used incur a $250 \mathrm{~ms}$ delay on the data, which can be penalizing for real-time applications. Table I mentions "hp" for a high-pass filter where the $0 \mathrm{~Hz}$ component is nullified, and a "bp" filter with at least $-50 \mathrm{~dB}$ attenuation at $0 \mathrm{~Hz}$ and above $50 \mathrm{~Hz}$, in each case with less than $1 \mathrm{~dB}$ change in the $6-30 \mathrm{~Hz}$ band.

Only 2 subjects out of the 13 subjects considered here are common with [5]. Our results both contrast with and confirm some points of [5]. One conclusion there is that Yule-Walker estimation of an auto-regressive model, which is then used to estimate the power density spectrum, is adequate for BCI tasks. In our experiments this technique performed well only when pre-filtering the data with a low or band-pass filter. It globally turns as the second-best technique $(3.8 \%$ below the maximum accuracy on average) with a relative stability (3.4\% standard deviation) of that performance, ex-æquo with the standard Spectrogram estimation. The Spectrogram leads here to a a better stabililty ( $3 \%$ standard deviation), but given the low number of subjects to perform the statistical analysis both methods seem equally suited.

However our results also contrast with [5]. They noticed that "Morlet CWT produced clearly the least separable feature" amongst the category of features they considered. On the contrary, we here find that the Morlet wavelet produces very good results, better than the auto-regressive model technique on the test sets, and directly on the raw data $(2.6 \%$ below the maximal accuracy on average vs $3.8 \%$, and $2.4 \%$ vs $3 \%$ for the stability). This difference is probably explained by the way we use the wavelet. In [5] the approach is to pre-tune the wavelet time-frequency resolution in order to concentrate on specific frequency $\mu$ and $\beta$ bands a priori, and only keep the extracted values at these frequencies. In the present study we cross-validate the wavelet time-frequency resolution to adapt it to the data, and we keep all frequencies available for the classifier. Thus we claim that with adequate pre-processing, the Morlet wavelet shall be considered as a technique of choice 


\begin{tabular}{|c|c|c|c|c|c|c|c|c|c|c|c|c|c|c|c|c|c|c|}
\hline method & \multicolumn{3}{|c|}{ Periodogram } & \multicolumn{3}{|c|}{ Butterworth } & \multicolumn{3}{|c|}{ Auto-regressive } & \multicolumn{3}{|c|}{ Spectrogram } & \multicolumn{3}{|c|}{ Wigner-Ville } & \multicolumn{3}{|c|}{ Morlet wavelet } \\
\hline subjet & $\mathrm{hp}$ & $\mathrm{bp}$ & raw & $\mathrm{hp}$ & bp & raw & $\mathrm{hp}$ & bp & raw & $\mathrm{hp}$ & bp & raw & $\mathrm{hp}$ & bp & raw & $\mathrm{hp}$ & bp & raw \\
\hline bci2 & 78.6 & 74.3 & 72.1 & 77.1 & 72.9 & 78.6 & 70.7 & 76.4 & 75 & 78.6 & 82.1 & 80.7 & 75.7 & 76.4 & 77.9 & 73.6 & 74.3 & 77.1 \\
\hline s4 & 79.1 & 80.4 & 79.1 & 79.6 & 80.7 & 79.6 & 81.1 & 81.3 & 82.6 & 80.7 & 80.7 & 79.8 & 80.6 & 81.5 & 79.4 & 80.9 & 79.3 & 81.5 \\
\hline $\mathrm{x} 11$ & 75.9 & 77.4 & 75.7 & 74.8 & 75.9 & 76.3 & 78 & 76.1 & 76.9 & 78 & 79.3 & 79.1 & 77.6 & 77.2 & 77.6 & 79.1 & 78.5 & 80.4 \\
\hline OV & 93.6 & 92.1 & 91.1 & 93.9 & 91.8 & 48.2 & 91.8 & 84.3 & 46.1 & 95 & 89.6 & 49.3 & 93.9 & 87.5 & 49.6 & 93.6 & 90.4 & 92.9 \\
\hline bci4s1 & 70 & 72.2 & 71.2 & 72.8 & 71.9 & 72.8 & 67.8 & 72.5 & 72.5 & 70.3 & 71.6 & 71.2 & 72.2 & 72.2 & 71.9 & 75.9 & 73.8 & 77.5 \\
\hline bci4s2 & 51.8 & 57.9 & 55 & 56.1 & 57.5 & 52.1 & 58.6 & 52.5 & 52.1 & 58.2 & 57.5 & 55.7 & 52.5 & 53.2 & 51.8 & 55.7 & 60 & 56.4 \\
\hline bci4s3 & 57.2 & 51.6 & 54.4 & 55.6 & 55.6 & 54.7 & 55.6 & 56.2 & 55.3 & 55.3 & 53.1 & 53.1 & 53.4 & 57.8 & 51.9 & 46.6 & 47.2 & 51.9 \\
\hline bci4s4 & 94.1 & 94.1 & 94.1 & 93.8 & 92.2 & 94.1 & 93.1 & 95.3 & 93.1 & 93.4 & 94.1 & 93.8 & 88.8 & 93.8 & 90 & 92.8 & 93.8 & 93.4 \\
\hline bci4s5 & 84.7 & 84.1 & 85 & 85 & 86.9 & 87.5 & 94.7 & 85.3 & 92.8 & 86.2 & 86.2 & 85.3 & 85 & 85.6 & 85 & 92.2 & 91.2 & 96.9 \\
\hline bci4s6 & 85.3 & 84.1 & 84.4 & 85.6 & 85.6 & 85.3 & 85.3 & 83.8 & 85 & 86.2 & 86.6 & 83.8 & 83.8 & 85.3 & 82.5 & 87.2 & 78.4 & 87.8 \\
\hline bci4s7 & 72.2 & 70.9 & 67.5 & 71.2 & 69.4 & 69.7 & 74.1 & 71.6 & 69.7 & 67.2 & 65.3 & 65.3 & 68.1 & 69.7 & 63.8 & 72.5 & $\begin{array}{l}73.4 \\
\end{array}$ & 70.6 \\
\hline bci4s8 & 83.1 & 84.4 & 79.4 & 84.4 & 84.4 & 83.4 & 81.6 & 82.5 & 82.2 & 81.9 & 81.6 & 83.8 & 82.2 & 81.9 & 82.5 & 81.9 & 85.3 & 80 \\
\hline bci4s9 & 80.3 & 83.4 & 79.4 & 78.4 & 78.4 & 79.4 & 77.8 & 84.7 & 76.6 & 79.4 & 79.1 & 79.4 & 81.9 & 82.2 & 81.2 & 81.6 & 80 & 78.8 \\
\hline $\begin{array}{l}\text { avg } \\
\delta \max \end{array}$ & 4.12 & 4.05 & 5.47 & 3.94 & 4.33 & 7.52 & 3.79 & 4.38 & 7.66 & 3.78 & 4.05 & 7.63 & 4.91 & 4.25 & 8.8 & 3.53 & 4.15 & 2.64 \\
\hline $\begin{array}{l}\text { dev } \\
\delta m a x\end{array}$ & 3.34 & 3.33 & 2.86 & 2.95 & 2.8 & 12.02 & 3.41 & 3.72 & 12.61 & 2.96 & 3.21 & 11.85 & 2.86 & 3.08 & 11.36 & 3.1 & 3.47 & 2.36 \\
\hline
\end{tabular}

Bold figures indicate the maximum accuracy for each subject, gray background results within $1.5 \%$ to the maximum. "hp" stands for highpass prefiltering of the data (see the main text), "bp" indicates a band-pass filtering, and "raw" represents the unfiltered data. $\delta m a x$ is the difference to the maximum accuracy obtained for each subject.

Table I

CLASSIFICATION ACCURACY (\%) OF POWER EXTRACTION TECHNIQUES ON THE TEST DATA SETS

for extracting power information for BCI tasks.

\section{CONCLUSION}

This study has reproduced and extended the comparisons performed in [5] to identify the best method for extracting the power in given frequency bands, for BCI purposes. We have evaluated the features obtained with the various band power estimation methods on motor imagery data from several EEG data sets, including the effect of pre-filtering the data. Our results both confirm and contrast with [5]. We confirmed that using the Yule-Walker algorithm for estimating an autoregressive model is a good technique for estimating the power in selected frequency bands for BCI tasks. But we have found, unlike [5], that the Morlet wavelet seems better suited. This difference between the two comparative studies is probably due to our cross-validation of the Morlet wavelet parameters and keeping more frequency bands.

In any case the Butterworth filter, commonly used in the litterature [1], is here amongst the worse techniques. We thus stress as in [5] that better power estimation algorithms shall be considered for BCI tasks. Software for computing all the features we used is widely available on the Internet [12]. The code for all the experiments performed in this study is also provided on the main author's web page ${ }^{4}$.

\section{ACKNOWLEDGMENTS}

This work has been partially supported by the French National Research Agency within the "OpenViBE2" project.

\footnotetext{
${ }^{4}$ http://nicolas.brodu.numerimoire.net/
}

\section{REFERENCES}

[1] G. Pfurtscheller and C. Neuper, "Motor imagery and direct braincomputer communication," proceedings of the IEEE, vol. 89, no. 7, pp. 1123-1134, 2001

[2] G. Pfurtscheller, G. Muller-Putz, R. Scherer, and C. Neuper, "Rehabilitation with brain-computer interface systems," IEEE Computer, vol. 41, pp. 58-65, 2008.

[3] A. Bashashati, M. Fatourechi, R. K. Ward, and G. E. Birch, "A survey of signal processing algorithms in brain-computer interfaces based on electrical brain signals," Journal of Neural engineering, vol. 4, no. 2, pp. R35-57, 2007.

[4] P. Flandrin, Temps-Fréquence. Hermès, Paris, 1998.

[5] P. Herman, G. Prasad, T. M. McGinnity, and D. Coyle, "Comparative analysis of spectral approaches to feature extraction for eeg-based motor imagery classification," IEEE Transactions on Neural Systems and Rehabilitation Engineering, vol. 16, no. 4, August 2008.

[6] G. Pfurtscheller, C. Brunner, R. Leeb, R. Scherer, G. Müller-Putz, C. Neuper, A. C. Elitzur, M. P. Silverman, J. Tuszynski, R. Vaas, H. D. Zeh, and B. Graimann, The Graz Brain-Computer Interface BrainComputer Interfaces. Springer Berlin Heidelberg, 2010.

[7] G. Pfurtscheller, G. R. Müller-Putz, A. Schlögl, B. Graimann, R. Scherer, R. Leeb, C. Brunner, C. Keinrath, F. Lee, G. Townsend, C. Vidaurre, and C. Neuper, "15 years of bci research at graz university of technology: Current projects," IEEE Transactions on Neural System and Rehabilitation Engineering, no. 14, pp. 205-210, 2006.

[8] B. Blankertz, G. Dornhege, M. Krauledat, G. Curio, and K.-R. Müller, "The non-invasive berlin brain-computer interface: Fast acquisition of effective performance in untrained subjects," NeuroImage, no. 37, pp. 539-550, 2007.

[9] V. Attina, E. Maby, R. Bouet, G. Gibert, J. Mattout, and O. Bertrand, "The importance of individual features for motor-imagery based bci," in 4th International Brain-Computer Interface Workshop and Training Course, 2008.

[10] F. Lotte, M. Congedo, A. Lécuyer, F. Lamarche, and B. Arnaldi, "A review of classification algorithms for eeg-based brain-computer interfaces," Journal of Neural Engineering, vol. 4, pp. R1-R13, 2007.

[11] Y. Renard, F. Lotte, G. Gibert, M. Congedo, E. Maby, V. Delannoy, O. Bertrand, and A. Lécuyer, "Openvibe: An open-source software platform to design, test and use brain-computer interfaces in real and virtual environments," Presence: teleoperators and virtual environments, vol. 19 , no. $1,2010$. 
[12] F. Auger, P. Flandrin, P. Gonçalvès, and O. Lemoine, "The timefrequency toolbox tutorial," CNRS (France), Rice University (USA), Tech. Rep., 1995-1996.

[13] N. Brodu, "Multifractal feature vectors for brain-computer interfaces," in IJCNN, 2008, pp. 2883-2890.

[14] B. Blankertz, K. R. Müller, G. Curio, T. M. Vaughan, G. Schalk, J. R. Wolpaw, A. Schlögl, C. Neuper, G. Pfurtscheller, T. Hinterberger, M. Schröder, and N. Birbaumer, "The BCI competition 2003: Progress and perspectives in detection and discrimination of EEG single trials," IEEE Transactions on Biomedical Engineering, vol. 51, no. 6, pp. 1044 1051,2004

[15] B. Blankertz, K. R. Muller, D. J. Krusienski, G. Schalk, J. R. Wolpaw, A. Schlogl, G. Pfurtscheller, J. D. R. Millan, M. Schroder, and N. Birbaumer, "The BCI competition III: Validating alternative approaches to actual BCI problems," IEEE Transactions on Neural Systems and Rehabilitation Engineering, vol. 14, no. 2, pp. 153-159, 2006.
[16] S. A., N. C., and P. G., "Estimating the mutual information of an eegbased brain-computer-interface," Biomedizinische Technik, vol. 47, no. 1-2, pp. 3-8, 2002.

[17] S. Lemm, C. Schafer, and G. Curio, "BCI competition 2003-data set III: probabilistic modeling of sensorimotor mu rhythms for classification of imaginary hand movements," IEEE Transactions on Biomedical Engineering, vol. 51, no. 6, pp. 1077-1080, 2004.

[18] B. Hjorth, "An on-line transformation of eeg scalp potentials into orthogonal source derivations." Electroencephalography and Clinical Neurophysiology, vol. 39, pp. 526-530, 1975.

[19] D. J. McFarland, L. M. McCane, S. V. David, and J. R. Wolpaw, "Spatial filter selection for EEG-based communication," Electroencephalographic Clinical Neurophysiology, vol. 103, no. 3, pp. 386-394, 1997. 